\title{
BMJ Open Early Parenting Acceptance and Commitment Therapy 'Early PACT' for parents of infants with cerebral palsy: a study protocol of a randomised controlled trial
}

\author{
Koa Whittingham (D) , ${ }^{1}$ Jeanie Sheffield, ${ }^{2}$ Catherine Mak (D) , ${ }^{1}$ Corrine Dickinson, ${ }^{1}$ \\ Roslyn N Boyd ${ }^{1}$
}

To cite: Whittingham $\mathrm{K}$, Sheffield J, Mak C, et al. Early Parenting Acceptance and Commitment Therapy 'Early PACT' for parents of infants with cerebral palsy: a study protocol of a randomised controlled trial. BMJ Open 2020;10:e037033. doi:10.1136/ bmjopen-2020-037033

- Prepublication history and additional material for this paper are available online. To view these files, please visit the journal online (http://dx.doi. org/10.1136/bmjopen-2020037033).

Received 17 January 2020 Revised 19 August 2020 Accepted 25 August 2020

Check for updates

(c) Author(s) (or their employer(s)) 2020. Re-use permitted under CC BY-NC. No commercial re-use. See rights and permissions. Published by BMJ.

${ }^{1}$ Queensland Cerebral Palsy and Rehabilitation Research Centre, UQ Child Health Research Centre (CHRC), Faculty of Medicine,

The University of Queensland, Brisbane, Queensland, Australia ${ }^{2}$ School of Psychology, The University of Queensland, Saint Lucia, Queensland, Australia

Correspondence to Dr Koa Whittingham; koawhittingham@uq.edu.au

\section{ABSTRACT}

Introduction New international clinical practice guidelines exist for identifying infants at high risk of cerebral palsy (CP) earlier: between 12 to 24 weeks corrected age, significantly earlier than previous diagnosis windows in Australia at 19 months. The earlier detection of infants at high risk of $\mathrm{CP}$ creates an opportunity for earlier intervention. The quality of the parent-infant relationship impacts various child outcomes, and is leveraged in other forms of intervention. This paper presents the protocol of a randomised controlled trial of an online parent support programme, Early Parenting Acceptance and Commitment Therapy (Early PACT) for families of infants identified as at high risk of CP. We predict that participating in the Early PACT programme will be associated with improvements in the parent-infant relationship, in parent mental health and well-being as well as infant behaviour and quality of life.

Methods and analysis This study aims to recruit 60 parents of infants ( 0 to 2 years old corrected age) diagnosed with CP or identified as at high risk of having CP. Participants will be randomly allocated to one of two groups: Early PACT or waitlist control (1:1). Early PACT is an online parent support programme grounded in Acceptance and Commitment Therapy (ACT). It is delivered as a course on an open source course management system called edX. Early PACT is designed to support parental adjustment and parent-infant relationship around the time of early diagnosis. Assessments will be conducted at baseline, following completion of Early PACT and at 6-month follow-up (retention). The primary outcome will be the quality of parent-child interactions as measured by the Emotional Availability Scale. Standard analysis methods for randomised controlled trial will be used to make comparisons between the two groups (Early PACT and waitlist control). Retention of effects will be examined at 6-month follow-up.

Ethics and dissemination This study is approved through appropriate Australian and New Zealand ethics committees (see in text) with parents providing written informed consent. Findings from this trial will be disseminated through peer-reviewed journal publications and conference presentations.

\section{Strengths and limitations of this study}

- To our knowledge this is one of the first randomised controlled trials (RCT) designed to look at supporting parents of infants newly diagnosed with cerebralpalsy (CP) or identified as having a high risk of CP with a preventative care programme, complementing the new international clinical guidelines for the early diagnosis of $\mathrm{CP}$.

- This RCT uses the Consolidated Standards of Reporting Trials methodology.

- The primary outcome is a parent-infant observation coded by blinded coders.

- The use of online delivery offers parents greater access and flexibility to EarlyParenting Acceptance and Commitment Therapy (Early PACT). The Early PACT course has the potential to be disseminated widely, in an accessible manner at minimal cost. The waitlist control condition enables all participants to have access to Early PACT over the course of their active participation for ethical reasons.

Trial registration details This trial has been prospectively registered on 12 June 2018 to present (ongoing) with the Australian New Zealand Clinical Trials Registry (ACTRN12618000986279); https://www.anzctr.org.au/ Trial/Registration/TrialReview.aspx?id=374896

\section{INTRODUCTION}

Earlier diagnosis and impact of cerebral palsy Diagnosis of cerebral palsy (CP) in Australia has traditionally commonly occurs in the first 24 months of life. ${ }^{1}$ New international clinical practice guidelines for identifying infants at high risk of CP aim to shift detection earlier: to 12 to 24 weeks corrected age. ${ }^{2-6}$ Earlier detection of infants at high risk of CP both creates an opportunity for earlier intervention and raises questions around the effect of early detection and intervention on the developing parent-child relationship. In this 
changing clinical context, it is important to explore pathways to supporting parental adjustment and the parentchild relationship in this early stage, from the point of diagnosis/identification as high risk.

Following the diagnosis of a neurodevelopmental disorder such as CP, parents grieve ${ }^{7}$ and many desire therapeutic support. ${ }^{8}$ The grieving process varies from parent to parent, but there is often a period of intense grief at the beginning with intensified grieving during major life events. ${ }^{7}$ Grief includes not just sadness but also anxiety about the future. ${ }^{9}$ Parents of children with CP are at risk for poorer psychological adjustment compared with mothers of typically developing children ${ }^{10-12}$ including depressive and anxious symptoms, ${ }^{13} 14$ parental stress ${ }^{15}$ and reduced quality of life. ${ }^{11}$ Parents of infants at high risk of CP need to establish a happy and healthy parentchild bond, ${ }^{16}$ but may need support to achieve this. ${ }^{8}$

\section{Parental responsiveness and child development}

The parent-child relationship in the first year of life is foundational to child development. Responsive, emotionally available parenting impacts on multiple infant outcomes, including attachment, psychological adjustment, social behaviour and cognitive development including for children with disabilities. Responsive, emotionally available parenting is child-directed, contingent and appropriate to the child's needs and development. From a child development perspective, responsive parenting enriches the environment of the infant, enabling the stimulationhungry infant brain to obtain the optimal 'dose' of stimulation for neurological development. ${ }^{17}$

\section{Existing interventions are limited for parents of infants identified as high-risk for $\mathrm{CP}$}

A systematic review identified no interventions targeting parental adjustment and well-being designed specifically for parents of infants with CP or identified as at high-risk of CP. ${ }^{18}$ The review identified research of interventions in parents of infants born preterm had a moderate effect for reducing symptoms of trauma and stress immediately after intervention with longer-term reductions in depression and anxiety symptoms. There were no significant differences in efficacy between intervention frameworks across studies. cognitive-behavioural therapy (CBT) was the most commonly represented approach. The variation in intervention dosage, framework and delivery pose a challenge in identifying effective elements when developing early interventions for parents of high-risk infants. Future randomised controlled trials (RCTs) are required to investigate: a broader neurodevelopmental risk population, outcomes for parents, infants and the parent-infant dyad, to explore trauma in the earlier diagnosis time frame and to provide longer-term follow-up post-intervention.

\section{Acceptance and commitment therapy}

Acceptance and Commitment Therapy (ACT) is part of the CBT family of therapies and aims to enhance psychological flexibility: the ability persevere or alter your behaviour with full awareness of your present-moment experience and context, based on your chosen values. ${ }^{19}$ As such, ACT emphasises the importance of values or intrinsically rewarding patterns of behaviour, present-moment awareness or mindfulness and experiential acceptance or ongoing non-reactive, open and receptive contact with psychological events such as difficult emotions, cognitions and memories.

ACT has a growing evidence base for a range of conditions and populations, ${ }^{20-23}$ including parenting. ${ }^{24-27}$ This has included trials for parents of children with neurodevelopmental disabilities such as acquired brain injury and autistic spectrum disorder. ${ }^{25}{ }^{26}$ In the CP population in particular, the combination of an established parenting intervention with ACT was shown to improve parental adjustment, child behaviour and child quality of life. ${ }^{28} 29$

The current study is the trial of EarlyParenting Acceptance and Commitment Therapy (Early PACT), an online ACT parenting support package, specifically tailored to families of infants ( $<2$ years old) diagnosed with $\mathrm{CP}$ or identified as high risk of $\mathrm{CP}^{16}{ }^{16}$ It is a tailored version of Parenting Acceptance and Commitment Therapy (PACT), an online parental support package trial with families of young children (2 to 10 years old) diagnosed with $\mathrm{CP}^{16}$ The adaptation of PACT was undertaken grounded on qualitative research with parents who received an early diagnosis for their infant as high risk for CP (Dickinson, Sheffield, Boyd and Whittingham, Parent experiences of their infant's diagnosis of risk of cerebral palsy: a qualitative study), as well as with health professionals who work with families of children with CP (Dickinson, Sheffield, Boyd and Whittingham, Understanding health professionals' perspectives of the impact of earlier diagnosis of infants at risk of cerebral palsy: a brief report).

\section{Aim/objectives}

The aim of this study is to demonstrate the effectiveness of a preventative online early parenting support package: Early PACT for families of infants $(<36$ months corrected age) with CP in supporting the parent-child relationship, parental adjustment and well-being and child adjustment and quality of life. Outcomes will be assessed immediately post-intervention and at 6 months post-intervention (retention).

\section{Hypotheses}

We predict that compared with waitlist control, Early PACT will result in:

1. Improved emotional availability as measured by the Emotional Availability Scales (EAS) ${ }^{30} 31$ and the Emotional Availability-Self Report (EA-SR). ${ }^{32}$

2. Improved parental adjustment as measured by the Acceptance and Action Questionnaire (AAQ-7), ${ }^{33}$ PTSD Checklist for DSM-5 (PCL-5) ${ }^{34}$ and improved parental well-being as measured by Personal Well-being Index (PWI). ${ }^{35}$ 
3. Improved infant adjustment as measured by Ages and Stages Questionnaire: Social-Emotional Version 2 (ASQ:SEv2) ${ }^{36}$ and quality of life as measured by The Infant Toddler Quality of Life Questionnaire (ITQOLSF47) ${ }^{37}$

4. In addition, we predict that: Early PACT will be feasible and acceptable to families.

\section{METHOD \\ Ethics}

Full ethical approval for this study has been obtained by the University of Queensland's Human Research Ethics Committees (2018001076), the Children's Health Queensland Hospital and Health Service Research Ethics Committee (HREC/18/QRCH/82) and the New Zealand Health and Disability Ethics Committees (19/CEN/72). Any protocol modifications and amendments will be submitted to the ethical committees for approval. This trial has been registered with the Australian New Zealand Clinical Trials Registry (ACTRN12618000986279). All parents will be asked to provide written informed consent to participate before entering into the trial.

\section{Design}

This study is a RCT following CONSORT (Consolidated Standards of Reporting Trials) guidelines. Parents of infants aged $<36$ months corrected age with a diagnosis of $\mathrm{CP}$ or identified as high risk of CP will be randomly allocated to one of two groups:

1. Early PACT in the form of the online edX course Early PARENT101.

2. Waitlist control (usual-care).

The waitlist control group will commence Early PACT after a 3-month waiting time. This design will allow researchers to compare Early PACT to a waitlist control group receiving usual-care alone and enable all participating parents to receive the intervention, maximising participant recruitment and retention, and fulfilling ethical obligations to participating families. Follow-up will occur at 6 months post-intervention (retention).

\section{Patient and public involvement}

As mentioned earlier, the development of Early PACT was undertaken grounded on qualitative research with parents who received an early diagnosis for their infant as high risk for CP, as well as with health professionals who work with families of children with CP (Dickinson, Sheffield, Boyd and Whittingham, Parent experiences of their infant's diagnosis of risk of cerebral palsy: a qualitative study; and Dickinson, Sheffield, Boyd and Whittingham, Understanding health professionals' perspectives of the impact of earlier diagnosis of infants at risk of cerebral palsy: a brief report). No patients were involved in the recruitment to or conduct of this study protocol. Patient self-referral and word of mouth will assist with recruitment for the RCT. In addition, patient feedback will be collected qualitatively via survey and a semi-structured interview as their experiences and opinion will inform the suitability of offering the Early PACT course as an early support option to parents around the time of their infants' diagnosis. The results of the RCT will be disseminated to study participants via research centre e-newsletters, as well as through peer-reviewed publications and national and international conferences.

\section{Participants and recruitment}

Eligible participants in this study will be parents of infants ( $<36$ months corrected age) diagnosed with $\mathrm{CP}$ or identified/diagnosed as at high risk of having CP by an appropriately qualified clinician, or based on the results of the General Movements or Hammersmith Infant Neurological Examination assessments, in accordance with the latest recommendations around early detection. ${ }^{2}$ Early PACT is being trialled as an online course, therefore, parents are requested to have:

1. Access to reliable Internet access (eg, ADSL) and be committed to maintaining Internet access for the duration of the intervention (10 weeks) and

2. An email address for receiving emails that they are committed to checking throughout the study.

Parents who do not fit the above criteria will be excluded from the study. Parents of infants with comorbidities will be included into the study.

Participants will be recruited through the Queensland Cerebral Palsy and Rehabilitation Research Centre, the early detection networks of the Australasian Cerebral Palsy Clinical Trials Network, Queensland Early Detection and Intervention Network - Cerebral Palsy, Cerebral Palsy Alliance (NSW) and The University of Auckland, New Zealand, and word of mouth. Early PACT uses online delivery and all of the assessments are also performed online so participants can be recruited from across Australia and New Zealand, including regional areas, making this project highly accessible. In hospitals, relevant clinicians will develop recruitment protocols that introduce the study details to eligible families and refer interested families to the study. In other settings, study flyer and promotional letters with contact details of the researchers will be disseminated to eligible participants. A referral form has been developed in either hard copy or online format for ease and convenience to assist clinicians in identifying and referring potentially eligible families. Parents can also self-refer via this form. The form requests contact details for the family so the Early PACT study coordinator can contact them and assess study eligibility further, explain the study in detail to parents and obtain consent to participate. The online form also has a link to the Early PACT study flyer, so clinicians only require one link for information about the study when they are meeting with families in their usual clinics. Enrolment for Early PACT commenced in March 2019.

\section{Sample size}

Various factors were considered in determining an appropriate sample size. Previous studies have found mean differences between intervention and control 
groups on these subscales from tele-interventions for parents in range of 0.4 to $1.5 .^{38}$ The present study would like to detect effect sizes within this range $(0.7)$ with a statistical power of 0.8 . Accounting for baseline scores, it is assumed a correlation between pre-intervention and post-intervention outcomes is 0.5 (as per, ${ }^{39}$ to detect a 0.7 effect size with 0.05 significance level and 0.8 statistical power, a sample size of 48 participants is required (24 in each group, generated by Sample Size Calculator from ClinCalc.com). Accounting for an $80 \%$ retention rate of participants we plan to recruit 60 participants. This is feasible as Early PACT intervention and assessments are delivered online, so, recruitment can include parents throughout Australia and New Zealand. Families of infants identified as 'high risk of CP' will be readily recruited using the early detections networks of the Australasian CP Clinical Trials Network.

\section{Randomisation}

After families have expressed their interest in participating in the study, they will be contacted by researchers to assess their eligibility for participation. Participation in the study is voluntary. Written informed consent (online supplemental appendix 1) will be sought prior to commencement. Following consent, participants will be asked to complete baseline assessments before being randomly allocated to either the intervention or the waitlist control group.

Randomisation and data collected in this study will be managed on the University of Queensland's (UQ) secure Research Electronic Data Capture (REDCap) platform. REDCap is a secure web platform designed to build and manage online databases and surveys. A computer-generated fixed block size randomisation with allocation ratio of $1: 1$, will be used to generate a random allocation order. An individual outside of the study (UQ REDCap administrator) will upload this into REDCap's randomisation function. The original file containing the random allocation order will be deleted. Following this, REDCap will generate each participant's group allocation when prompted by the researchers after the participant has completed their baseline assessments. Although the researchers will not be involved in the randomisation process, due to the study design, they will not be blinded to participants' condition status.

Families allocated to the Early PACT intervention group will receive access to the online course for immediate enrolment, while families allocated to the waitlist control group will be advised of their group allocation and timelines. In the interests of equity and retaining participants, participants in the waitlist control group will be offered access to the Early PACT course after completion of the post-intervention review assessment. The experimental design and outcome measures are illustrated in figure 1.

Study personnel will check-in with participants at different times when they are completing the Early PACT course online and will monitor participants' adherence to the course. Study personnel will also problem solve

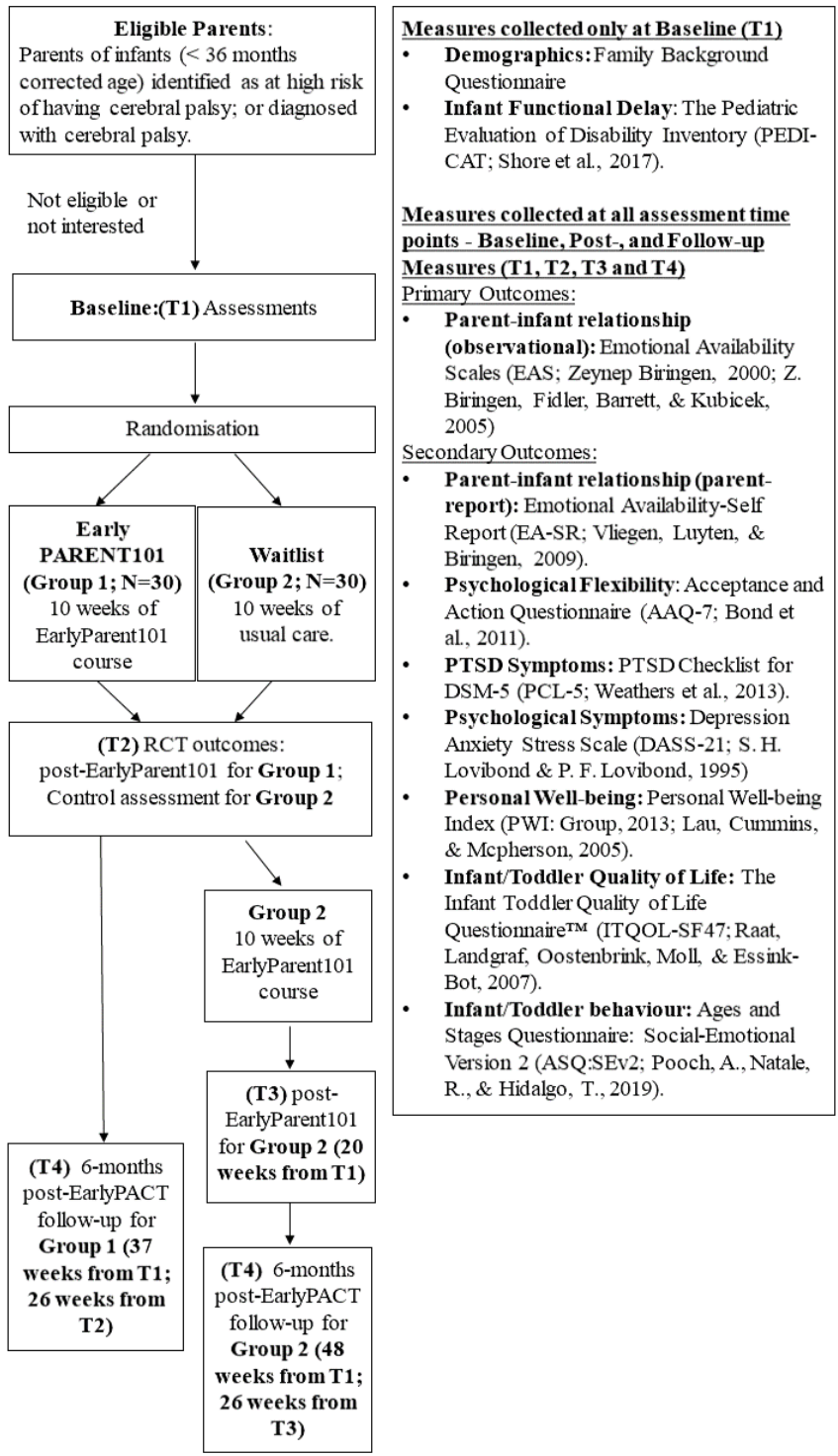

Figure 1 Flowchart of Early PACT study design. Early PACT, EarlyParenting Acceptance and Commitment Therapy; PTSD, post-traumatic stress disorder; RCT, randomised controlled trial.

with participants in relation to any barriers to adhering to or completing the intervention online as intended.

\section{Intervention}

Early PACT is a parenting course adapted from PACT, a parenting intervention previously designed for families of children (2 to 10 years of age) with CP. ${ }^{16}$ Both Early PACT and PACT are grounded in the ACT approach, ${ }^{19}$ with the aim of enhancing the parent-infant relationship and parental emotional responsiveness. The Early PACT course was created in the edX platform and has been named Early PARENT101. It consists of video presentations, virtual text information, interactive activities, reflections and journal activities, as well guided experiential exercises to promote mindfulness and selfcompassion. Parents will also be able to participate in a 
moderated discussion board with other parent participants. Early PARENT101 comprises of three modules plus a review session. Each module consists of approximately 2 to 3 hours of content and exercises, and course design allows parents flexibility to move through it at their own pace. Each module is scheduled to be completed in a fortnight. After the third module, there is a 4-week break to allow for practice of the materials from the course. The whole course will take a total of 10 weeks to complete. The course content will explore parents' values, thoughts and feelings, mindfulness techniques, ways to meet their infant's needs and exercises to develop compassion. Parents will be able to watch video presentations and engage in exercises to reflect on what they have learnt and how they might practice the strategies with their own child.

To maximise participant retention and support engagement in the Early PARENT101 course, email or text message reminders will be sent to participants to complete the online assessments and approximately five phone or Zoom consultations will be conducted by study personnel with each participant throughout completion of the course. These consultations aim to facilitate and support engagement in the course, as well as to assist with the individuasation and adaptation of the strateiges from Early PARENT101 to participants' everyday life.

\section{MEASURES}

\section{Baseline measures}

Family Background Questionnaire

This is tailored from existing measures to assess family factors and demographic variables such as socio-economic status, parental education and family factors specific to the CP context. Some questions include: 'What is your highest education level?' with checkbox options ranging from 'Primary school or less' to 'Post-graduate (Masters/ Doctorate) equivalent' and 'Please indicate age of your child when he/she was diagnosed as at risk of having cerebral palsy (months)' with checkbox options ranging from 0 to 36 months.

\section{The Paediatric Evaluation of Disability Inventory, Computer Adaptive Test}

This is designed for use from birth to 20 years of age to identify functional delay across three domains: Daily Activities, Mobility and Social/Cognitive. It has been shown to have good discriminant validity in CP populations. ${ }^{40}$

\section{Primary outcome measures}

Can Early PACT optimise the parent-infant relationship?

Emotional Availability Scales

A 20 min naturalistic observation of the parent-infant relationship will be scored using the EAS. ${ }^{3031}$ The interaction will be by the research team via Internet-connected video recording (Zoom). The EAS measures the quality of the relationship itself across six scales: parental sensitivity, parental structuring, parental non-intrusiveness, parental non-hostility child responsiveness and child involvement and it generates an overall relationship quality rating. The scale has high inter-rater reliability for parental responsiveness $(0.96)$, involving $(0.87)$, sensitivity $(0.93)$ and structuring (0.76).

\section{Secondary outcome measures}

Can Early PACT optimise the parent-infant relationship per parent self-report?

\section{Emotional Availability-Self Report}

This is a 32-item parent-report measure of emotional availability within the parent-child relationship. ${ }^{32}$ It has excellent reliability and validity. The EA-SR produces five subscales: mutual attunement, affect quality, capacity to involve the parent, intrusiveness and hostility.

\section{Can Early PACT improve parental adjustment and well-being? Acceptance and Action Questionnaire}

This is a 7-item measure of psychological inflexibility and experiential avoidance, that is, attempts to control or avoid thoughts, memories and emotions, particularly when doing so causes harm. It produces a single total scale with high scores reflecting high psychology inflexibility and experiential avoidance. The AAQ has satisfactory internal consistency $(\alpha=0.79){ }^{33}$

\section{PTSD Checklist for DSM-5}

This is a 20-item self-report measure that screens individuals for post-traumatic stress disorder (PTSD) according to DSM-5 symptoms. ${ }^{34}$ The measure asks the individual to rate how each symptom statement impacted them in the last month on a scale ranging from $0=$ not at all, $1=\mathrm{a}$ little bit, $2=$ moderately, $3=$ quite a bit and $4=$ extremely. A total symptom severity score (ranging from 0 to 80 ) is obtained with a score of 33 or above considered a positive screen for PTSD symptoms.

\section{Depression Anxiety Stress Scale}

This is a 21-item self-report questionnaire. ${ }^{41}$ It measures symptoms of depression, anxiety and stress in adults. Participants rate items on a 4-point Likert scale, reflecting how much the statement applied to them over the past week. Each of the three subscales in the DASS have good internal consistency: the depression $(\alpha=0.91)$, anxiety $(\alpha=0.84)$ and stress $(\alpha=0.90)$ scales. The DASS also has good discriminant and concurrent validity. ${ }^{43}$

\section{Personal Well-being Index}

This is $\mathrm{s}$ an 8-item measure of personal well-being in adults. Participants rate items on a 10-point Likert scale, reflecting their degree of satisfaction with various demands of living. The ratings for each of the eight items are summed to create an average score representing subjective well-being participants can also be asked to rate their life as a whole. The Well-being Index has good validity and internal consistency $(\alpha>0.170) .^{3544}$ 
Can PACT optimise child behaviour, adjustment and quality of life? The Infant Toddler Quality of Life Questionnaire

The ITQOL was developed for use in infants and toddlers of 2 months to 5 years age. ${ }^{37}$ The ITQOL short form measures quality of life across physical, mental and social well-being. The test has 47 items in the short-form and is completed by parent-report. For each of the 47 concepts, item responses are scored, summed and transformed to a scale from 0 (worst health) to 100 (best health).

\section{Ages and Stages Questionnaire: Social-Emotional Version 2}

The ASQ:SEv2 is designed to screen children's behaviours in seven areas: self-regulation, compliance, adaptive function, autonomy, affect, social-communication and interaction with people. ${ }^{45}$ These behavioural challenges are known comorbidities with cerebral palsy.

\section{What do parents think of Early PACT?}

Satisfaction with Early PACT content and format will be measured using a questionnaire specifically developed for this study. The questionnaire will ask participating parents to rate various aspects of Early PACT on 10-point scale as well as give qualitative feedback. A secondary goal of this study is to determine if Early PACT would be suitable for clinicians to offer parents at the time a diagnosis or a high risk of $\mathrm{CP}$ is communicated to parents. A blinded independent interviewer, will therefore collect some qualitative information by conducting a semi-structured phone interviews with parents individually post-intervention to gain an understanding parents' experience around the disclosure of diagnosis, their experience of the Early PACT course and their opinion on whether or not it would be a suitable support option offered to parents around the time of diagnosis.

\section{Data analysis}

Analyses will follow standard principles for RCTs using two-group comparisons on all participants on an intention-to-treat basis. Primary comparison immediately post-intervention (T2) based on EAS will be between treatment groups using analysis of covariance with treatment group (Early PACT/waitlist control) included as the main effect and baseline EAS as the covariate. Effect estimates will be presented as mean difference and 95\% CI. Secondary outcome analyses will use similar methods to compare all other outcomes between groups immediately post-intervention (T2). Similar outcome analyses will also be used to compare outcomes for secondary parent (second parent from the same household) between groups. In cases where interval data are not able to be transformed appropriately for regression analyses, nonparametric methods (Mann-Whitney U test) will be used for between-treatment comparisons. Possible differential attrition will be assessed by comparing baseline characteristics of drop-outs and continuing participants using t-tests (or Mann-Whitney U test) for continuous variables and $\chi^{2}$ tests for categorical variables. Sensitivity analyses of all outcomes will be conducted using multiple imputation techniques, to investigate the effect of non-ignorable missing data during follow-up. A senior biostatistician will provide advice for guiding and assisting with the analysis. Data will be analysed using SPSS.

\section{Qualitative data analysis}

The qualitative data collected in this study will be analysed using a descriptive thematic analysis. ${ }^{46}$ The semi-structured interviews will be audio-recorded and transcribed verbatim. Transcripts will be coded by two study investigators. Thematic patterns such as ideas, concepts, terminology or phrases will be identified during the coding process. The investigators will discuss the appropriateness of the identified themes and develop a mutual coding framework until consensus is achieved. The researchers will then organise the themes into categories and subcategories based on the coding framework they have developed.

\section{Data management}

In this study, all questionnaire data will be collected and managed on the University of Queensland's (UQ) secure REDCap platform. The specific REDCap platform used in this study is hosted on UQ's secure server. All the questionnaire responses provided by the participants will be confidential and participants will not be identified by name. Data will be automatically saved on the secure REDCap platform within the UQ server.

Video recordings of parent-child interactions will be recorded on Zoom by the research team at UQ. The videos will be saved on the secure UQ server immediately after the recording session is complete. Video file names will be in de-identified but re-identifiable form. Any data published will be completely de-identified so as not to reveal the identity of any participant. Signed consent forms will be scanned and saved as digital files and research data including video footage will be stored in electronic form within folders only accessible to study personnel on a University of Queensland secure server.

Data including recordings will be retained in accordance with the obligations under the Public Records Act 2002 and associated State policies, retention and disposal schedules and other official advice issued by the Queensland State Archives' State Archivist https://www. qld.gov.au/dsiti/qsa.

\section{Ethical considerations}

All parents will be requested to provide informed consent to participate prior to entering into the trial. Participants will be reminded before they commence the programme that they can choose not to engage in any content and that they can withdraw from the study at any point without prejudice. Should parents be identified throughout the trial as experiencing psychological distress that warrants professional input, advice on referral to local support options will be provided. A Data Monitoring Committee is not considered necessary. The chief investigators will review any adverse events or unintended effect detected. 


\section{Dissemination of results}

This trial has been prospectively registered with the Australian New Zealand Clinical Trials Registry (ACTRN12618000986279). Findings from this research will be disseminated through the broader scientific community via publication in peer-reviewed journals and via presentation at conferences. If found successful we can make Early PACT accessible to parents of newly diagnosed infants across the world.

\section{DISCUSSION}

This study aims to evaluate whether Early PACT, a flexible and easily translatable online course, grounded in ACT will be effective at improving parent-infant relationships for families with an infant identified as at high risk of CP. If found effective Early PACT could provide immediate support to parents of newly diagnosed infants.

Acknowledgements Thank you to the parent participants and health professionals who shared their experiences and provided advice to the development of Early PACT course and study (Dickinson, Sheffield, Boyd and Whittingham, Parent experiences of their infant's diagnosis of risk of cerebral palsy: a qualitative study; and Dickinson, Sheffield, Boyd and Whittingham, Understanding health professionals' perspectives of the impact of earlier diagnosis of infants at risk of cerebral palsy: a brief report). Thank you to Professor Robert Ware for his expert statistical advice and guidance in the design of this study.

Contributors KW took a lead role in designing this study. CD conducted semistructured interviews with families and synthesised findings to assist with the adaptation of PACT to Early PACT. KW, JS and CD worked together, created the edX course Early PARENT101. CM managed the randomised controlled trial and contributed to drafts of the protocol. JS, CD and RNB both contributed their expertise on study design and contributed to drafts of the protocol.

Funding This work was supported by a Cerebral Palsy Alliance Grant (PG11917) and a National Health and Medical Research Council postdoctoral fellowship (KW 631712) and a National Health and Medical Research Council fellowship (RB 1105038).

Competing interests None declared.

Patient and public involvement Patients and/or the public were involved in the design, or conduct, or reporting, or dissemination plans of this research. Refer to the Methods section for further details.

Patient consent for publication Not required.

Provenance and peer review Not commissioned; externally peer reviewed.

Open access This is an open access article distributed in accordance with the Creative Commons Attribution Non Commercial (CC BY-NC 4.0) license, which permits others to distribute, remix, adapt, build upon this work non-commercially, and license their derivative works on different terms, provided the original work is properly cited, appropriate credit is given, any changes made indicated, and the use is non-commercial. See: http://creativecommons.org/licenses/by-nc/4.0/.

\section{ORCID iDs}

Koa Whittingham http://orcid.org/0000-0002-5344-9907

Catherine Mak http://orcid.org/0000-0001-7446-2767

\section{REFERENCES}

1 Australia Cerebral Palsy Register. Australian cerebral palsy register report 2018. Canberra: The Australian Cerebral Palsy Register Group, 2018.

2 Novak I, Morgan C, Adde L, et al. Early, accurate diagnosis and early intervention in cerebral palsy: advances in diagnosis and treatment. JAMA Pediatr 2017;171:897-907.

3 Bosanquet M, Copeland L, Ware R, et al. A systematic review of tests to predict cerebral palsy in young children. Dev Med Child Neurol 2013;55:418-26.
4 Spittle AJ, Doyle LW, Boyd RN. A systematic review of the Clinimetric properties of neuromotor assessments for preterm infants during the first year of life. Dev Med Child Neurol 2008;50:254-66.

5 Romeo DMM, Cioni M, Palermo F, et al. Neurological assessment in infants discharged from a neonatal intensive care unit. Eur $\mathrm{J}$ Paediatr Neurol 2013;17:192-8.

6 Romeo DM, Ricci D, Brogna C, et al. Use of the Hammersmith infant neurological examination in infants with cerebral palsy: a critical review of the literature. Dev Med Child Neurol 2016;58:240-5.

7 Whittingham K, Wee D, Sanders MR, et al. Sorrow, coping and resiliency: parents of children with cerebral palsy share their experiences. Disabil Rehabil 2013;35:1447-52.

8 Dagenais L, Hall N, Majnemer A, et al. Communicating a diagnosis of cerebral palsy: caregiver satisfaction and stress. Pediatr Neurol 2006;35:408-14.

9 Murray J. Understanding loss: a guide for caring for those facing adversity. London: Taylor and Francis, 2015.

10 Cheshire A, Barlow JH, Powell LA. The psychosocial well-being of parents of children with cerebral palsy: a comparison study. Disabil Rehabil 2010;32:1673-7.

11 Pousada M, Guillamón N, Hernández-Encuentra E, et al. Impact of caring for a child with cerebral palsy on the quality of life of parents: a systematic review of the literature. J Dev Phys Disabil 2013;25:545-77.

12 Michael O, Olufemi O, Jasola F, et al. Psychosocial problems among mothers of children with cerebral palsy attending physiotherapy outpatient department of two selected tertiary health centres in Ogun state: a pilot study, 2019.

13 Barlow JH, Cullen-Powell LA, Cheshire A. Psychological well-being among mothers of children with cerebral palsy. Early Child Dev Care 2006; $176: 421-8$.

14 Lach LM, Kohen DE, Garner RE, et al. The health and psychosocial functioning of caregivers of children with neurodevelopmental disorders. Disabil Rehabil 2009;31:741-52.

15 Rentinck ICM, Ketelaar M, Jongmans MJ, et al. Parents of children with cerebral palsy: a review of factors related to the process of adaptation. Child Care Health Dev 2007;33:161-9.

16 Whittingham K, Sheffield J, Boyd RN. Parenting acceptance and commitment therapy: a randomised controlled trial of an innovative online course for families of children with cerebral palsy. BMJ Open 2016;6:e012807.

17 Mills-Koonce WR, Willoughby MT, Zvara B, et al. Mothers' and fathers' sensitivity and children's cognitive development in lowincome, rural families. J Appl Dev Psychol 2015;38:1-10.

18 Dickinson C, Whittingham K, Sheffield J, et al. Efficacy of interventions to improve psychological adjustment for parents of infants with or at risk of neurodevelopmental disability: a systematic review. Infant Ment Health J 2020;1:1-26.

19 Hayes SC. Acceptance and Commitment Therapy and the new behaviour therapies. In: Hayes SC, Follette VM, Linehan MM, et al, eds. Mindfulness and acceptance expanding hte cognitive-behavioral tradition. New York: The Guilford Press, 2004.

20 Griffiths C, Williamson $\mathrm{H}$, Zucchelli $\mathrm{F}$, et al. A systematic review of the effectiveness of acceptance and commitment therapy (act) for body image Dissatisfaction and weight Self-Stigma in adults. J Contemp Psychother 2018;48:189-204.

21 Twohig MP, Levin ME. Acceptance and commitment therapy as a treatment for anxiety and depression: a review. Psychiatr Clin North Am 2017;40:751-70.

22 Hughes LS, Clark J, Colclough JA, et al. Acceptance and commitment therapy (act) for chronic pain: a systematic review and meta-analyses. Clin J Pain 2017;33:552-68.

23 Swain J, Hancock K, Dixon A, et al. Acceptance and commitment therapy for children: a systematic review of intervention studies. $J$ Contextual Behav Sci 2015;4:73-85.

24 Whittingham K, Coyne L. Acceptance and Commitment Therapy the Clinician's Guide for Supporting Parents. San Diego: Academic Press, 2019.

25 Hahs AD, Dixon MR, Paliliunas D. Randomized controlled trial of a brief acceptance and commitment training for parents of individuals diagnosed with autism spectrum disorders. J Contextual Behav Sci 2019;12:154-9.

26 Lunsky $\mathrm{Y}$, Fung $\mathrm{K}$, Lake $\mathrm{J}$, et al. Evaluation of acceptance and commitment therapy (act) for mothers of children and youth with autism SpectrumDisorder. Mindfulness 2018;9:1110-6.

27 Whittingham K. Becoming mum: pivotal publishing, 2015.

28 Whittingham K, Sanders M, McKinlay L, et al. Interventions to reduce behavioral problems in children with cerebral palsy: an RCT. Pediatrics 2014;133:e1249-57. 
29 Whittingham K, Sanders M, McKinlay L, et al. Stepping stones triple $\mathrm{P}$ and acceptance and commitment therapy for parents of children with cerebral palsy: trial protocol. Brain Impairment 2013;14:270-80.

30 Biringen Z. Emotional availability: conceptualization and research findings. Am J Orthopsychiatry 2000;70:104-14.

31 Biringen Z, Fidler DJ, Barrett KC, et al. Applying the emotional availability scales to children with disabilities. Infant Ment Health J 2005;26:369-91.

32 Vliegen N, Luyten P, Biringen Z. A Multimethod perspective on emotional availability in the postpartum period. Parenting 2009;9:228-43.

33 Bond FW, Hayes SC, Baer RA, et al. Preliminary psychometric properties of the acceptance and action Questionnaire-II: a revised measure of psychological inflexibility and experiential avoidance. Behav Ther 2011;42:676-88.

34 Weathers F, Litz B, Keane T, et al. Ptsd checklist for DSM-5 (PCL-5), 2013.

35 International Wellbeing Group. Personal wellbeing index. 5th Edn. Melbourne: Australian Centre on Quality of Life, Deakin University, 2013.

36 Squires J, Bricker D, Twombly E. The ASQ: SE user's guide: For the Ages \& Stages Questionnaires: Social-emotional: Paul H Brookes Publishing, 2002.

37 Raat H, Landgraf JM, Oostenbrink R, et al. Reliability and validity of the infant and toddler quality of life questionnaire (ITQOL) in a general population and respiratory disease sample. Qual Life Res 2007;16:445-60.
38 Baker M, Biringen Z, Meyer-Parsons B, et al. Emotional attachment and emotional availability tele-intervention for adoptive families. Infant Ment Health J 2015;36:179-92.

39 Walters SJ, Jacques RM, Dos Anjos Henriques-Cadby IB, et al. Sample size estimation for randomised controlled trials with repeated assessment of patient-reported outcomes: what correlation between baseline and follow-up outcomes should we assume? Trials 2019;20:566.

40 Shore BJ, Allar BG, Miller PE, et al. Evaluating the discriminant validity of the pediatric evaluation of disability inventory: computer adaptive test in children with cerebral palsy. Phys Ther 2017;97:669-76.

41 Lovibond SH, Lovibond PF. Manual for the depression anxiety stress scales. 2nd. Ed. Sydney: Psychology Foundation, 1995.

42 Lovibond PF, Lovibond SH. The structure of negative emotional states: comparison of the depression anxiety stress scales (DASS) with the Beck depression and anxiety inventories. Behav Res Ther 1995;33:335-43.

43 Brown TA, Chorpita BF, Korotitsch W, et al. Psychometric properties of the depression anxiety stress scales (DASS) in clinical samples. Behav Res Ther 1997;35:79-89.

44 ALD L, Cummins RA, Mcpherson W. An investigation into the cross-cultural equivalence of the personal wellbeing index. Social Indicators Research 2005;72:403-30.

45 Pooch A, Natale R, Hidalgo T, Ages HT. Ages and stages questionnaire: Social-Emotional as a Teacher-Report measure. J Early Interv 2019;41:3-12.

46 Braun V, Clarke V. Using thematic analysis in psychology. Qual Res Psychol 2006;3:77-101. 